\title{
Travail original
}

\section{Méthodologie de l'enquête MISSION (Ménopause, rISque de cancer du Seln, mOrbidité et prévaleNce)}

\author{
T. Chevallier*, J.-P. Daurès*, M.-C. Micheletti ${ }^{\star *}$, J.-Y. Reginster ${ }^{\star * *}$ \\ et le groupe MISSION ${ }^{\star \star \star \star}$
}

* Laboratoire de Biostatistique, d'Épidémiologie et de Recherche Clinique, I.U.R.C., 641, avenue du Doyen-Giraud, 34000 Montpellier.

** Laboratoire Théramex, 6, avenue du Prince-Héréditaire-Albert, 98007 Monaco.

*** Centre Collaborateur de l'O.M.S. (Organisation Mondiale de la Santé) pour les aspects de Santé Publique des affections rhumatismales et de l'ostéoporose, C.H.U. Centre Ville, Quai Godefroid-Kurth, 45, Bat K1, 4020 Liège, Belgique.

**** Groupe MISSION : Présidents des Collèges de la Fédération Nationale des Collèges de Gynécologie Médicale (F.N.C.G.M.), Pia de REILHAC (Ouest) : coordinatrice, Suzanne DAT (Midi), Jean DeRRIEN (Rhônes-Alpes), Solange Hugues-BoetTo (Marseille Provence),

Françoise Mousteou (Sud-Est), Elizabeth PAGANELLI (Centre et Val-de-Loire), Isabelle PagNIEZ (Nord), Paul EVAngelIStI (Bordeaux et Sud-Ouest), Dominique SCHICKELE-PIERRE (Lorraine et Région Est), David SERFATY (Paris, Ile-de-France), Jean-Louis SiMENEL (Normandie).

\begin{abstract}
RÉSUMÉ
Objectif. Cet article a pour ambition de détailler et d'expliquer la méthodologie de l'enquête MISSION (Ménopause : risque de cancer du sein, morbidité et prévalence) dont les buts sont de déterminer en France la prévalence du cancer du sein et de la morbidité hors cancer du sein, chez la femme ménopausée suivie par un gynécologue et traitée par un Traitement Hormonal Substitutif (THS) ou non.

Matériel et méthode. Six mille six cents femmes ménopausées [3300 sous THS — à l'étude uniquement : pour l'œstrogène : œstradiol par voie orale ou cutanée ; pour le progestatif associé le cas échéant : progestérone naturelle et assimilés ou l'un des dérivés prégnanes (à l'exception de spécialités contenant du médroxyprogestérone acétate) ou nor-prégnanes - et 3300 sans THS] seront recrutées en France par 825 gynécologues investigateurs membres ou non de la Fédération Nationale des Collèges de Gynécologie Médicale (FNCGM) du 5 janvier 2004 au 28 février 2005. II s'agit d'une enquête de type historico-prospective avec tirage au sort des cas recrutés. Elle est menée à l'initiative du Laboratoire ThéramexMerck sous l'égide d'un Centre Collaborateur de l'O.M.S. (Organisation Mondiale de la Santé) pour les aspects de Santé Publique des affections rhumatismales et de l'ostéoporose et en collaboration avec un groupe d'experts multidisciplinaires. Conclusion. Les premiers résultats contribueront à l'amélioration de nos connaissances en santé de la femme et apporteront des éléments propres à un hypothétique particularisme à la française sur le relationnel entre THS et cancer du sein.
\end{abstract}

Mots-clés : Ménopause • Morbidité • Épidémiologie • Enquête • Traitement hormonal substitutif.

SUMMARY: Mission methodology: menopause, breast cancer risk, morbidity and prevalence.

Objective. The ambition of this article is to detail and to explain the methodology of the study named MISSION (Menopause: breast cancer risk, morbidity and prevalence). The aims of MISSION are to determine the prevalence of breast cancer and global morbidity in France among menopausal women treated or not with hormone replacement therapy (HRT) and followed by a gynecologist.

Material and method. 6600 menopausal women [3300 with HRT — ie for estrogen: only estradiol via oral or transdermal administration; for progestogen: natural progesterone or assimiled or one pregnane derived (excluding medroxyprogesterone acetate) or non-pregnane derived — and 3300 without HRT] will be enrolled in France between January 52004 and February 282005 by 825 gynecologists, members or not of the National Federation of Medical Gynecologists (FNCGM).

This study design is a historico-prospective with case randomization.

MISSION is conducted by a Theramex-Merck Laboratories initiative in collaboration with a WHO (World Health Organization) Collaborating Center for Public Health Aspects of Rheumatic Diseases and a multidisciplinary expert group.

Conclusion. First results of this study will contribute to better knowledge of women health.

Key words: Menopause • Morbidity • Epidemiology • Study • Hormone replacement therapy.

Tirés à part : M.-C. Micheletti, à l'adresse ci-dessus. E-mail : mcmicheletti@theramex.mc

Reçu le 15 février 2005. Avis du Comité de Lecture le 26 mai 2005. Définitivement accepté le 23 juin 2005.

(c) MASSON, Paris, 2005. 
L'étude des facteurs de risque du cancer du sein a, depuis longtemps, mis en cause le système hormonal en montrant une association entre ce risque et la puberté précoce, l'âge tardif de la première grossesse, la ménopause tardive et le faible nombre d'enfants [1].

La relation entre le risque de cancer du sein chez la femme ménopausée et le Traitement Hormonal Substitutif (THS) de la ménopause est soumise à controverse, notamment depuis les récentes publications de la WHI (Women Health Initiative) [2] et de la MWS (Million Women Study) [3] qui n'ont pourtant que globalement confirmé les acquis relativement stables et rassurants à ce sujet, suite à la méta-analyse d'Oxford de 1997 [4].

La controverse repose, entre autres, sur la difficulté à extrapoler, à la France, les résultats issus de ces études anglo-saxonnes et ce, pour deux principales raisons : les caractéristiques des populations étudiées sont très différentes de celles de la France (âge, et Indice de Masse Corporelle notamment) ; les molécules disponibles en France et prises par la grande majorité des femmes sous THS ne sont pas évaluées dans ces études.

L'état de l'art des études intégrant ces particularismes à la française et le relationnel entre THS et cancer du sein est le suivant: l'étude européenne WISDOW (méthodologiquement comparable à la WHI) utilisant des molécules «européennes » dont les résultats étaient très attendus, a été interrompue par manque de volontaires suite à l'arrêt prématuré de la WHI ; nous ne disposons pas en France, malgré l'existence de registre de cancer (FRANCIM notamment) de données directes concernant l'incidence et/ ou la prévalence du cancer du sein, spécifiquement chez la femme ménopausée, qu'elle soit traitée ou non et permettant notamment de servir de base de référence ; les seules données sur l'incidence et/ou la prévalence du cancer du sein disponibles en France, sont présentées par tranche d'âge sans précision sur le statut ménopausique et la prise (actuelle ou passée) ou non d'un THS [5-7]; très récemment, la publication de Fournier et al. [8] sur les premiers résultats de la cohorte E3N a apporté des éléments tout à fait intéressants, laissant subodorer que le risque de cancer du sein vis-à-vis du THS serait très dépendant du type de progestatif utilisé (notamment progestérone naturelle versus progestatif synthétique).

À l'heure actuelle, selon les données de l'étude WHI [2] on estime que pour 1000 femmes sous placebo, environ 16 cas de cancer du sein invasifs seront diagnostiqués en 5 ans de suivi et que, pour 1000 femmes utilisant une association œstrogène/ progestatif, le nombre de cas supplémentaires serait, selon la meilleure estimation de 4, pour 5 ans d'utilisation (Monographie Vidal 2004).

Toutefois, le manque de données propres à la situation française demeure un sujet sensible et préoccupant pour la majorité des gynécologues cliniciens et prescripteurs de THS. La prescription de THS actuellement en France devrait, a priori, s'appuyer sur les récentes recommandations de l'AFSSAPS [9].

C'est dans ce contexte que l'enquête MISSION (Ménopause : rISque de cancer du SeIn, mOrbidité et prévaleNce) a été mise en place depuis le 5 janvier 2004, en collaboration avec la Fédération Nationale des Collèges de Gynécologie Médicale (FNCGM), et sous l'égide d'un Centre Collaborateur de l'OMS (Organisation Mondiale de la Santé) pour les aspects de Santé Publique des affections rhumatismales et de l'ostéoporose, afin de contribuer à combler ce manque de données propres à la situation française, en répondant à un double objectif :

- d'une part, déterminer la prévalence en France du cancer du sein, chez la femme ménopausée suivie par un gynécologue et traitée par THS ou non, notamment en fonction de la durée d'utilisation et du type de ce THS (objectif principal) ;

- d'autre part, déterminer la prévalence en France de la morbidité hors cancer du sein (fractures de type ostéoporotique, infarctus du myocarde, accident vasculaire cérébral, phlébite, embolie pulmonaire, lithiase vésiculaire, incontinence urinaire, autres cancers, autres événements...) chez la femme ménopausée suivie par un gynécologue et traitée par THS ou non, notamment en fonction de la durée d'utilisation et du type du THS (objectif secondaire).

Dans un second temps, l'enquête MISSION aura pour objectif, sur la cohorte de femmes ménopausées constituée, de déterminer l'incidence des différentes pathologies sus-citées.

Cet article a pour ambition de détailler et d'expliquer la méthodologie de cette enquête prospective de grande envergure.

\section{PATIENTES ET MÉTHODE}

La Fédération Nationale des Collèges de Gynécologie Médicale (FNCGM), créée en janvier 1984, comprend actuellement 2035 adhérents répartis en 11 collèges (Bordeaux et Sud-Ouest, Centre et Val- 
de-Loire, Lorraine et régions Est, Marseille-Provence, Midi, Nord, Normandie, Ouest, Paris - Ilede-France, Rhône-Alpes, Sud-Est).

Huit cent vingt-cinq gynécologues appartenant prioritairement à l'un des collèges sus-cités, se sont portés volontaires bénévoles pour participer à cette enquête et au recrutement des patientes. Ce nombre total de gynécologues a été ventilé en fonction de la taille initiale du collège, elle-même dépendante de la taille de la région, afin de garantir une représentativité nationale dans le mode de recrutement (tableau I).

Chaque gynécologue doit recruter 8 cas patientes (4 cas traités et 4 cas non traités), ce qui porte, en considérant les 825 investigateurs, à 6600 (3 300 cas traités et 3300 cas non traités) le total de cas patientes à recruter.

Ce nombre de sujets requis s'appuie sur les règles calculatoires suivantes: risque de $1^{\text {re }}$ espèce : $\alpha=0,05$; risque de $2^{\mathrm{e}}$ espèce : $\beta=0,05$; puissance : 1 - $\beta=0,95$ (soit $95 \%$ ) ; valeur de $\phi$ en hypothèse unilatérale : $\phi=\left(\mathrm{Z}_{\alpha}-\mathrm{Z}_{1-\beta}\right)=3,290$; SMR (Standardized Morbidity Ratio) à 5 ans estimé selon Nelson et al. $[10]=1,4$; calcul de $\mathrm{E}$ (nombre de cas attendus) : $E=\frac{\phi^{2}}{4[(\sqrt{S M R})-1]^{2}} ;$ estimation de l'incidence du cancer du sein chez la femme dans la population générale dans la tranche d'âge 45-64 ans [5] : I = 250 /100 000 ; soit R le recul moyen fixé à 5 ans pour cha-

Tableau I Répartition des gynécologues investigateurs par collège de la FNCGM.

Distribution of the gynaecologist investigations by FNCGM college.

\begin{tabular}{lc}
\hline \multicolumn{1}{c}{ Collège } & $\begin{array}{c}\text { Nombre de gynécologues } \\
\text { par collège }\end{array}$ \\
\hline Bordeaux et Sud-Ouest & 93 \\
Centre et Val-de-Loire & 40 \\
Lorraine et Régions Est & 48 \\
Marseille - Provence & 62 \\
Midi & 73 \\
Nord & 62 \\
Normandie & 75 \\
Ouest & 82 \\
Paris - Île-de-France & 193 \\
Rhône-Alpes & 62 \\
Sud-Est & 35 \\
\hline Total & 825 \\
\hline
\end{tabular}

que patiente incluse, le nombre total théorique de sujets à inclure est $: \mathrm{N}=\frac{\mathrm{E}}{\mathrm{R}^{*} \mathrm{I}}=6448,80$. Le recrutement des cas patientes pour chaque médecin investigateur repose sur un double aléa (fig. 1): le premier correspond au tirage au sort du «jour d'inclusion » : il y a ainsi, par médecin, 4 « jours d'inclusion » cas traités et 4 « jours d'inclusion» cas non traités. Ce tirage au sort des «jours d'inclusion» n'est pas stratifié sur le mois, ni sur les jours de la semaine, ni sur l'activité en terme de volume de consultation du gynécologue; le deuxième correspond à la sélection de la patiente proprement dite : il repose par « jour d'inclusion » sur une suite de 10 lettres alphabétiques tirées au sort; le «jour d'inclusion » le médecin inclut la première patiente «cas » dont le nom usuel (marital ou de jeune fille) commence par une de ces lettres en respectant leur ordre de classement. En cas « d'inclusion blanche » i.e. aucun recrutement possible dû aux contraintes imposées, des couples « jour inclusion-lettre alphabétique » supplémentaires sont fournis au fur et à mesure, au médecin. En pratique chaque médecin a, en sa possession, une liste de randomisation comportant le jour d'inclusion et la liste aléatoire des lettres alphabétiques. Pour chaque jour d'inclusion, le médecin doit inclure la première patiente de la journée dont le nom usuel commence par la première lettre de la série alphabétique. Si aucune patiente ne correspond aux critères, il doit inclure la première patiente de la journée dont le nom commence par la deuxième lettre de la série alphabétique et ainsi de suite... Une liste de secours est disponible et n'est à utiliser que lorsque la liste initiale n'a pu être respectée (absence du médecin, pas de patiente correspondant aux critères d'inclusion...).

Pour être incluse, une patiente «cas traité » doit répondre aux critères suivants: être ménopausée naturellement (diagnostic porté sur l'existence chez une femme de 45 ans ou plus d'une aménorrhée supérieure ou égale à 12 mois et/ou l'existence de 3 tests au progestatif négatifs) ou chirurgicalement; être traitée actuellement ou avoir été traitée par THS (celui-ci ayant été arrêté depuis moins de 5 ans) quelle que soit l'antériorité de ce traitement, à condition que celui-ci soit ou ait été : - pour l'œstrogène : de type œstradiol par voie orale ou cutanée, — pour le progestatif associé le cas échéant : de la progestérone naturelle et assimilés ou l'un des dérivés de la 17$\mathrm{OH}$, de la 17-méthyl ou de la 19-nor-progestérone soit des dérivés prégnanes (à l'exception de spécialités contenant du médroxyprogestérone acétate) ou nor-prégnanes. 
Nom : xxx Prénom : xxx

Code postal : xxxx

Période :

Début : 12/1/2004

\section{Exclusion :}

lundis, vendredis, samedis, dimanches et jours fériés

\section{Numéro de centre : $\mathrm{xxx}$}

Fin : 13/3/2004

Liste Principale :

\begin{tabular}{|l|l|l|l|}
\hline Numéro de cas & Jour d'inclusion & Série alphabétique aléatoire * & Type de cas \\
\hline 1 & $14 / 01 / 2004$ & S V C P T G D Q R B & Non-Traitée \\
2 & $15 / 01 / 2004$ & E Z V O M B H R N Y & Non-Traitée \\
3 & $21 / 01 / 2004$ & B U I D Y C L S A J & Non-Traitée \\
4 & $22 / 01 / 2004$ & A Z O W P I C G L E & Traitée \\
5 & $29 / 01 / 2004$ & D U P O Z Q S G K C & Traitée \\
6 & $04 / 02 / 2004$ & X M U F S Y V E D P & Non-Traitée \\
7 & $11 / 02 / 2004$ & M X D U G E W A T Q & Traitée \\
8 & $18 / 02 / 2004$ & W D Z Q V A E G U R & Traitée \\
\hline
\end{tabular}

\section{Liste de secours :}

La liste de secours n'est à utiliser que lorsque la liste initiale n'a pu être respectée (absence, pas de patiente correspondant aux critères...). Il faut alors prendre le type de cas (Traitée, Non Traitée) correspondant au jour manqué.

\begin{tabular}{|l|l|l|l|}
\hline Numéro de cas & Jour d'inclusion & Série alphabétique aléatoire * & Type de cas \\
\hline 9 & $25 / 02 / 2004$ & V P Z Q K D T E W N & Traitée \\
10 & $26 / 02 / 2004$ & N X D H B L K F W Z & Non-Traitée \\
11 & $03 / 03 / 2004$ & Q Y O G B K H V U E & Traitée \\
12 & $11 / 03 / 2004$ & Z O K J L W G D M Y & Non-Traitée \\
\hline
\end{tabular}

Pour chaque jour d'inclusion, inclure la première patiente de la journée dont le nom usuel commence par la première lettre de la série alphabétique. Si aucune patiente ne correspond aux critères, inclure la première patiente de la journée dont le nom commence par la deuxième lettre de la série alphabétique et ainsi de suite...

Figure 1 Exemple de liste de randomisation. Example of the randomization list. 
Pour être incluse, une patiente « cas non traité » doit répondre aux mêmes critères que pour les patientes « cas traité » concernant le statut de ménopause ; en revanche, elle doit, soit ne jamais avoir pris de THS, soit l'avoir arrêté depuis au moins 5 ans et ce, quel que soit son type et la durée de la prise.

Pour chacun de ces 2 cas, le médecin investigateur doit relever au cours d'un interrogatoire simple et à l'aide d'un cahier d'observation dupliqué, respectant l'anonymat des patientes: des données anamnestiques et cliniques et des éventuels évènements sans aucune limite d'antériorité par rapport à l'ancienneté de la ménopause et du traitement hormonal (le cas échéant) : cancer du sein, fractures de type ostéoporotique, infarctus du myocarde, accident vasculaire cérébral, phlébite, embolie pulmonaire, lithiase vésiculaire, incontinence urinaire, autres cancers, autres évènements. Le recueil de ces évènements se fait historiquement sur dossier ou prospectivement s'il est un des motifs de consultation (recueil de cas prévalents et/ou incidents).

Le plan d'analyse statistique prévisionnel est le suivant - en analyse descriptive : caractéristiques des patientes incluses dans cette enquête ; taux de prévalence (et d'incidence annuelle et cumulée) des événements considérés dans cette enquête (cancer du sein et autres événements morbides) par grande catégorie (THS ou non); ventilation de ces taux en fonction du type de THS (selon la voie et le schéma d'administration, et le progestatif reçu), de la durée du THS et des caractéristiques patientes ; - en analyse inférentielle : comparaison des taux de prévalence (et d'incidence annuelle et cumulée) des différents événements considérés en fonction des différentes catégories de regroupement (THS ou non, type de THS, durée du THS, caractéristiques patientes...).

Tout médecin investigateur a la possibilité de rentrer en contact avec le promoteur par l'intermédiaire soit d'un numéro vert (0 8009125 99) soit par email (contact@enquetemission.com). Un site internet (www.etudemission.com) est également mis à leur disposition pour connaître, entre autres, l'état d'avancement de cette enquête.

Des actions de contrôle sont prévues dans le cadre du système d'assurance qualité pour vérifier que les exigences de qualité de l'enquête sont respectées et des audits internes seront effectués par l'Unité d'Assurance Qualité du promoteur de cette enquête.

Enfin, un comité de surveillance et d'éthique, dont le rôle est d'assurer le suivi de cette enquête et d'en garantir la validité éthique et scientifique, a été mis en place*.

\section{DISCUSSION}

L'état de l'art sur la relation entre le risque de cancer du sein chez la femme ménopausée et le THS de la ménopause peut être chronologiquement résumé ainsi :

— plus d'une soixantaine d'études observationnelles ont été publiées avec des résultats contradictoires ;

- la méta-analyse d'Oxford [4] utilisant plus de $90 \%$ des études publiées sur ce thème depuis 1997 (15 études de cohortes et 36 études cas-témoins soit un total de 53000 cancers du sein invasifs) a permis de conclure notamment que l'utilisation d'un THS par rapport à un groupe témoin se traduisait significativement par 2 cancers du sein supplémentaires pour une prise pendant 5 ans de ce traitement $[R R($ Risque Relatif $)=1,2$ ], 6 pour 10 ans $(R R=1,6)$ et 12 pour 15 ans $(\mathrm{RR}=2,2)$.

Quatre récentes études d'intervention sur les risques du THS ont pu apporter des éléments supplémentaires sur ce relationnel entre THS et cancer du sein :

- l'étude HERS [11] a mis en évidence une augmentation non significative du risque de cancer du sein chez des femmes ayant reçu une association ostro-progestative comparativement au placebo $(\mathrm{RR}=1,38$, Indice de Confiance à $95 \%=0,82-$ 2,31);

- l'étude WEST [12] a étudié l'effet des œstrogènes seuls comparativement au placebo. 5 cancers ont été relevés dans les 2 groupes lors des 2,8 ans de suivi ;

— l'étude PEPI [13] [875 femmes ménopausées randomisées entre placebo, œstrogènes conjugués équins $(\mathrm{ECE})+$ médroxyprogestérone acétate (MPA) ou $\mathrm{ECE}+$ progestérone micronisée] n'a pas montré entre les groupes, de différence en terme de risque de cancer du sein ;

- l'étude WHI [2], étude randomisée en double aveugle contre placebo menée chez 16000 femmes ménopausées a montré dans le groupe traité (association ECE + MPA), comparativement au groupe placebo un RR vis-à-vis du cancer du sein de 1,06 (IC95 $=0,81-1,38)$ pour des durées d'utilisation infé-

\footnotetext{
* Constitution du Comité d'Éthique et de Surveillance :

Coordinateur: Pr Pierre Mares, CHU Hôpital Caremeau (Nîmes). Collège d'experts : Dr Marc Espie, Hôpital Saint-Louis (Paris 10); Pr Thierry Maudelonde, Laboratoire de Biologie Cellulaire, CHU Hôpital Arnaud-deVilleuneuve (Montpellier); Pr Claude Sureau, Institut Théramex (Paris 16).
} 
rieure à 5 ans et de 2,13 (IC95 = 1,15-3,94) pour des durées d'utilisation supérieure ou égale à 5 ans et inférieure à 10 ans.

Depuis la WHI, de nombreuses publications sur ce sujet ont vu le jour et notamment :

- la méta-analyse de Béral et al. [14] qui va dans le même sens que la WHI avec un RR de cancer du sein sous THS estimé à 1,27 ;

- la méta-analyse de Nelson et al. [10] qui a estimé ce RR en fonction de la durée de prise totale d'un THS : pour une durée exacte de 5 ans de prise le RR se situe entre 1,21 et 1,40 , pour une durée de plus de 5 ans ce RR est compris entre 1,23 et 1,35. Il n'y a pas d'augmentation de ce RR pour une durée de prise d'un THS inférieure à 5 ans ;

- l'étude de Olsson et al. [15] de type observationnel, dont les conclusions ont relaté que le THS œstroprogestatif combiné continu augmentait de manière significative le risque de cancer du sein audelà de 4 ans d'utilisation, par rapport à l'absence de traitement (ceci n'étant pas observé avec le THS séquentiel) ;

- l'étude cas-témoin MWS (Million Women Study) [3] de type observationnel, où les auteurs ont observé que l'incidence du cancer du sein était augmentée chez les utilisatrices actuelles de THS avec un effet durée et ce, plus avec l'association d'un œstrogène et d'un progestatif que lors d'utilisation d'œstrogènes seuls ;

- l'étude rétrospective de B. de Lignières et al. [16] menée sur 3175 femmes ménopausées dont les conclusions sont peu extrapolables compte-tenu d'un biais de sélection important (recrutement sur un seul centre et uniquement hospitalier) et de l'étude du THS par voie cutanée uniquement, mais qui laisse suggérer que le risque relatif de cancer du sein est proche de 1 avec ce type de traitement lors d'un suivi de 8,9 ans ;

- récemment, les résultats finaux du bras œstrogènes seuls (ECE) de l'étude WHI [17] ont révélé de nouvelles contradictions : il n'a été observé aucune augmentation du risque de cancer du sein dans le groupe ECE comparativement au placebo, voire une tendance non significative à la baisse du nombre de cancers du sein, dans le groupe traité ;

- enfin, la très récente publication de Fournier et al. [8], sur la cohorte E3N a principalement montré :

- une légère augmentation significative du RR du cancer du sein chez les utilisatrices de THS (quel que soit le type) $(\mathrm{RR}=1,2)$;
- une absence d'augmentation significative de ce RR chez les femmes sous œstrogènes seuls $(\mathrm{RR}=1,1)$ ou sous association œstrogène (cutané) et progestérone micronisée $(\mathrm{RR}=0,9)$;

- une légère augmentation de ce risque chez les femmes sous œstrogènes et progestatifs synthétiques $(\mathrm{RR}=1,4)$, sans cependant distinguer les progestatifs synthétiques entre eux.

L'objectif de l'enquête MISSION est prioritairement de faire un état des lieux de la morbidité de la femme ménopausée avec ou sans THS pour contribuer à combler le manque de données cliniques propres à la situation française et c'est la seule en France qui pourra être en mesure de fournir des données directes, précises (car une stratification, notamment sur le type, la durée du THS, sera possible) et sur un échantillon dont la représentativité, a priori, peut être jugée satisfaisante.

Le déroulement de cette enquête a été dicté par des principes alliant à la fois des critères de rationnel scientifique, des critères méthodologiques propres aux enquêtes épidémiologiques et des critères de faisabilité et de coût.

La constitution d'un réseau de médecins investigateurs volontaires répartis équitablement sur toute la France n'est pas simple. Nous avons donc opté pour une collaboration avec la Fédération Nationale des Collèges de Gynécologie Médicale (FNCGM) pour plusieurs raisons : leur structure organisationnelle au niveau national intéressant la presque totalité des régions de France; leur mode de fonctionnement, avec à la tête de chaque collège un président et des réunions régulières de chacun des membres, nous permettant un échange de flux d'information.

Le volontariat reposant sur le bénévolat est une des clefs du caractère éthique de cette enquête car il n'y a aucun incitatif direct pour y participer. Certes, comme toute procédure faisant appel au volontariat, la notion de représentativité de l'échantillon des investigateurs peut être discutée. La situation «gold standard » pour contrecarrer cette entorse méthodologique aurait été de tirer au sort les médecins gynécologues et de leur proposer de participer bénévolement à cette enquête, puis de démontrer dans un deuxième temps que les volontaires ayant voulu participer n'étaient pas différents de ceux qui avaient refusé de participer à cette enquête après leur tirage au sort. Ceci, bien que conceptuellement très intéressant, n'est ni réalisable, ni déontologique.

Par ailleurs, il est certain qu'une des critiques qui sera, à tort ou à raison, faite à cette étude est le recru- 
tement par des gynécologues, praticiens de ville, dont on peut supposer au moins dans certaines villes, la tendance à ne pas suivre éventuellement leurs patientes atteintes de cancer du sein. La prévalence et l'incidence du cancer du sein peuvent être effectivement sous-évaluées par rapport à la population générale, mais, compte tenu du fait que le mode de recrutement est le même pour les «cas traités » et les « cas nontraités », cette tendance n'aura aucune action sur l'évaluation du sur risque potentiel attribuable notamment au THS. Une autre critique sur le mode de recrutement est possible : le nombre de cancers du sein dans le groupe non-traité peut être artificiellement supérieur à celui du groupe traité, compte tenu du fait que le cancer du sein est une contre-indication absolue au THS. Mais, cet effet peut être supposé comme faible pour la raison évoquée plus haut, du fait du recrutement par des gynécologues de ville qui ne suivent pas forcément les femmes ménopausées atteintes d'un cancer du sein. De plus, lors de la comparaison des deux groupes en termes de prévalence du cancer du sein, les femmes du groupe non-traité ayant un antécédent de cancer du sein avant ou après la ménopause seront exclues de l'analyse. D'une manière plus générale, gommer totalement ce genre de biais de sélection est en pratique très difficile. En effet, centre anti-cancéreux, centre de mammographie, CHU... sont autant de lieux facilitant le recrutement mais représentant chacun un biais de sélection. Même dans la WHI [2] (appel au volontariat par mailing direct et pour lequel d'ailleurs, la démonstration de la non-différence vis-à-vis du cancer du sein, des volontaires tirés au sort par rapport aux non-volontaires, fait défaut) et la MWS [3] (où les femmes fréquentaient un centre de dépistage mammographique) ces aspects de biais de sélection n'ont pas pu être évités.

Tel qu'est construit le protocole de l'enquête MISSION et notamment grâce à l'approche historicoprospective, les cas prévalents relevés seront documentés dans le temps (date de début du traitement, date d'apparition de la maladie, date de la ménopause). L'unité de mesure «personne-temps », prenant en compte le nombre de personnes atteintes par la maladie et la durée d'exposition à un facteur de risque supposé, sera donc possible à calculer.

La vision pragmatique prise en compte dans cette enquête est un élément important dans l'évaluation d'un risque lié à la prescription d'une thérapeutique donnée. En effet, la vision explicative et l'attribution randomisée d'un traitement dans cette évaluation ne peut pas être le reflet de la réalité car l'art de la pres- cription s'appuie toujours sur une estimation, de la part du prescripteur, de la balance bénéfice/risque de cette prescription pour un patient donné. Ainsi, dans la WHI [2], l'approche explicative randomisée bien qu'intellectuellement rassurante car normalisante, ne prend pas en compte cette dimension et des patientes qui du fait de la randomisation ont reçu un THS n'auraient pas été forcément candidates à ce traitement lors d'une prescription rationnelle car asymptomatiques par exemple. Enfin, notre méthodologie pragmatique nous met à l'abri des éventuelles difficultés de recrutement de patientes comme on a pu le constater dans l'approche explicative randomisée de la WISDOW, suite à la publication des premiers résultats de la WHI [2].

Le calcul du nombre de sujets nécessaires tient compte de la différence de fréquence vis-à-vis du cancer du sein selon la prise ou non de THS par l'intermédiaire du SMR qui, à 5 ans d'exposition, est estimé à 1,4 selon Nelson et al. [10]. Le choix de cette référence a été motivé par la nature de cette publication : scientific review et par l'absence de données propres à la situation française. Le risque de $1^{\text {re }}$ espèce a été fixé à $5 \%$ conformément aux standards requis en statistiques déductives. Le risque de $2^{\mathrm{e}}$ espèce a été fixé à $5 \%$ et permet d'assurer aux tests statistiques une puissance importante. Le recul $\mathrm{R}$ a été fixé à 5 ans car il semblerait que cela soit une durée d'exposition critique charnière en matière de risque attribuable au THS vis-à-vis du cancer du sein.

Un éventuel biais de recrutement est contrôlé par une procédure de tirage au sort à 2 niveaux, procédure qui est rigoureusement identique dans son application pour les 2 groupes. Toutefois notre procédure de tirage au sort n'a pas pris en compte a priori la notion d'activité du médecin au sens nombre de consultation de femmes ménopausées ce qui effectivement peut être considéré comme un biais de représentativité. Nous minimiserons ce biais a posteriori, au cours de l'analyse, par une pondération par l'activité du gynécologue au cours de la période de recrutement.

Le choix des traitements étudiés a été motivé par le contexte et l'état de l'art scientifique à ce sujet. Nous nous sommes centrés sur les THS «à la française » i.e. ceux les plus prescrits en France et non étudiés par la WHI [2] et la MWS [3], ou non distingués par leurs propriétés pharmacologiques, en ce qui concerne les progestatifs, dans le cadre de l'étude E3N [8].

Le questionnaire prend en compte les caractéristiques socio-démographiques de la patiente, les données de 
l'examen clinique, les antécédents médicaux et gynécologiques, etc. Les facteurs dits de «confusion» vis-à-vis du risque du cancer du sein, sont relevés dans le questionnaire de façon identique dans les 2 groupes pour une prise en compte, dans l'analyse en tant que variables d'appariement, lors de l'estimation du risque relatif.

Afin de garantir à cette enquête des modalités de «bonne pratique», nous avons nommé un comité d'éthique et de surveillance chargé d'assurer le suivi de cette enquête et d'en garantir la validité éthique et scientifique. Ce comité est composé de médecins indépendants, chacun excellant dans un domaine propre lui permettant au mieux de juger cette enquête.

\section{CONCLUSION}

À l'heure actuelle, la situation en France sur le relationnel entre THS et cancer du sein, reste floue. La récente publication de la cohorte $\mathrm{E} 3 \mathrm{~N}$ vient renforcer l'idée qu'il existe bien un particularisme « à la française » notamment par rapport aux molécules utilisées et enfonce le clou de ce qui a fait la genèse de MISSION, à savoir : les études anglo-saxonnes sont peu extrapolables à la situation française. Les premiers résultats de cette enquête MISSION, menée en collaboration avec la FNCGM, sont attendus avec impatience et devraient aller probablement dans le même sens que les premières conclusions de l'étude E3N, avec toutefois la possibilité de distinguer les progestatifs synthétiques selon leurs propriétés pharmacologiques.

\section{RÉFÉRENCES}

1. Clavel-Chapelon F, E3N Group. Cumulative number of menstrual cycles and breast cancer risk results from the E3N cohort study of French women. Cancer Causes Control 2002; 13: 831-8.

2. Writing Group for the Women's Health Initiative Investigators. Risks and benefits of estrogen plus progestin in healthy postmenopausal women. Principal results from the Women's
Health Initiative randomized controlled trial. JAMA 2002; 288: 321-33.

3. Million Women Study Collaborators. Breast cancer and hormone-replacement therapy in the Million Women Study. Lancet 2003; 362: 419-27.

4. Collaborative Group on Hormonal Factors in breast cancer. Breast cancer and hormone replacement therapy : collaborative reanalysis of data from 51 epidemiological studies of 52.705 women with breast cancer and 108.411 women without breast cancer. Lancet 1997; 350: 1047-59.

5. Direction Générale de la Santé. Cancer du sein. Rapport mars 2003.

6. Direction Générale de la Santé. Pathologies mammaires bénignes chez la femme. Rapport mars 2003.

7. Fédération Nationale des Observatoires Régionaux de la Santé. Rapport la santé observée dans les régions de France : Le cancer du sein 2000.

8. Fournier A, Berrino F, Riboli E, Avenel V, Clavel-Chapelon F. Breast cancer risk in relation to different types of hormone replacement therapy in the E3N-EPIC cohort. Int $J$ Cancer 2005; 114: 448-54.

9. AFSSAPS. Mise au point actualisée sur le Traitement Hormonal Substitutif de la ménopause (THS) - Décembre 2003.

10. Nelson HD, Humphrey LL, Nygren P, Teutsch SM, Allan JD. Postmenopausal hormone replacement therapy: scientific review. JAMA 2002; 288: 872-81.

11. Hulley S, Furberg C, Barrett-Connor E, Cauley J, Grady D, Haskell W et al. Noncardiovascular disease outcomes during 6.8 years of hormone replacement therapy: Heart and estrogen/progestin replacement study follow-up (HERS II). JAMA 2002; 288: 58-66.

12. Sancho-Garnier H. Epidemiology of breast cancer. Bull Acad Natl Med 1998; 182: 1621-32.

13. The Writing Group for the Pepi Trial. Effects of estrogen or estrogen/progestin regimen on heart disease risk factors in postmenopausal women. The postmenopausal estrogen/progestin interventions (PEPI) Trial. JAMA 1995; 273: 199-208.

14. Beral V, Banks $E$, Reeves $G$. Evidence from randomised trials on the long-term effects of hormone replacement therapy. Lancet 2002; 360: 942-44.

15. Olsson $\mathrm{HL}$, Ingvar $\mathrm{C}$, Bladstrom A. Hormone replacement therapy containing progestins and given continuously increases breast carcinoma risk in Sweden. Cancer 2003; 97: 1387-92.

16. De Lignières $B$, De Vathaire $F$, Fournier $S$, Urbinelli $R$, Allaert F, Le MG et al. Combined hormone replacement therapy and risk of breast cancer in a French cohort study of 3175 women. Climacteric 2002; 5: 332-40.

17. Anderson GL, Limacher M, Assaf AR, Bassford SA, Beresford SA, Black $\mathrm{H}$ et al. Effects of conjugated equine estrogen in postmenopausal women with hysterectomy : the Women's Heath Initiative randomized controlled trial. JAMA 2004; 291: 1701-12. 